\title{
The ROS controversy in hypoxic pulmonary hypertension revisited
}

\author{
Sébastien Bonnet ${ }^{1,2}$ and Olivier Boucherat ${ }^{1,2}$ \\ Affiliations: ${ }^{1}$ Pulmonary Hypertension Research Group, Centre de Recherche de l'Institut Universitaire de \\ Cardiologie et de Pneumologie de Québec, Université Laval, Québec, QC, Canada. ${ }^{2}$ Dept of Medicine,
} Université Laval, Québec, QC, Canada.

Correspondence: Sébastien Bonnet, Pulmonary Hypertension Research Group, IUCPQ Research Centre, 2725, chemin Sainte-Foy A2134, Québec, QC Canada, G1V 4G5. E-mail: sebastien.bonnetacriucpq.ulaval.ca

@ERSpublications

A study published in the ERJ investigates the role of superoxide in adaptation of the pulmonary vasculature to chronic hypoxia and adaptation of the right ventricle to increased afterload http://ow.ly/Dj9Q30iwO8R

Cite this article as: Bonnet S, Boucherat O. The ROS controversy in hypoxic pulmonary hypertension revisited. Eur Respir J 2018; 51: 1800276 [https://doi.org/10.1183/13993003.00276-2018].

Hypoxic pulmonary vasoconstriction (HPV) is a local and adaptive response to alveolar hypoxia causing constriction of small pulmonary arteries, thereby reducing perfusion to underventilated alveoli. However, in pathological conditions characterised by global and persistent hypoxia, such as chronic obstructive pulmonary disease (COPD), this physiological mechanism that normally serves to optimise ventilationperfusion matching become damaging, promoting sustained pulmonary vasoconstriction and vascular remodelling [1]. These structural changes are accompanied by a gradual increase of pulmonary arterial pressure, ultimately leading to right ventricular (RV) hypertrophy and failure. Although there is a global consensus amongst scientists that elevation of the intracellular calcium concentration plays a critical role in producing HPV, the precise underlying mechanisms (e.g. nature of the oxygen sensor, direction of changes in reactive oxygen species (ROS) as well as how ROS production affects the activity of calcium channels) and even the cell type involved remain a subject of ongoing debate [2-4].

Numerous studies have pinpointed the critical implication of ROS in HPV, but controversy remains regarding levels of ROS that mediate $\operatorname{HPV}[2,5]$. Due, at least in part, to the broad range of chemically distinct ROS, their highly reactive nature, as well as differing methods of quantification and models, conflicting results have been reported establishing two partly opposing models. In the first model so-called "redox hypothesis of HPV", the mitochondrial electron transport chain (ETC) senses hypoxia and decreases ROS production (ROS being produced as an inevitable byproduct of oxidative phosphorylation) causing intracellular calcium influx secondary to redox-sensitive potassium channels inhibition [6, 7]. In support of this, hypoxia and proximal ETC inhibitors have been found to suppress the production of activated $\mathrm{O}_{2}$ species and increase HPV in endothelium-denuded rings of distal pulmonary arteries [8] as well as isolated perfused rat lungs [6]. This model is challenged by the "ROS hypothesis", which proposes that hypoxia increases mitochondrial ROS generation mediating elevation of intracellular calcium concentration [9-11]. This theory is strengthened by studies showing that antioxidants such as catalase and glutathione peroxidase attenuated the HPV response [12] and exogenous $\mathrm{H}_{2} \mathrm{O}_{2}$ induces contraction in cultured pulmonary artery smooth muscle cells (PASMCs) and HPV in isolated lungs [13].

Received: Feb 062018 | Accepted after revision: Feb 132018

Conflict of interest: None declared.

Copyright OERS 2018 
In their study, PAK et al. [14] investigated the open question whether ROS are decreased or increased during acute and chronic hypoxia in the pulmonary vasculature and RV. Using complementary methods, the authors first documented changes in the redox state of mouse PASMCs during acute hypoxia $\left(1 \% \mathrm{O}_{2}\right.$ for $5 \mathrm{~min}$ ). They demonstrated that acute hypoxia induces an elevation of superoxide in PASMCs, which was prevented by a low dose of mitochondria-targeted antioxidant MitoQ exposure. Accordingly, levels of mitochondrial ROS assessed by MitoSOX in fluorescence microscopy and cytosolic $\mathrm{H}_{2} \mathrm{O}_{2}$ detected using the molecular sensor $\mathrm{HyPer}_{\text {cyto }}$ were upregulated in PASMCs subjected to acute hypoxia. In contrast, superoxide concentration in isolated lung fibroblasts exhibited an inverse fluctuation with a marked decreased in response to acute hypoxia. The investigators followed up on these observations using in vivo experiments. They showed that ROS levels are decreased in homogenates from intact lungs exposed to acute hypoxic ventilation, probably due to the fact that fibroblasts are present in a larger proportion.

In PASMCs exposed to chronic hypoxia ( $1 \% \mathrm{O}_{2}$ for 5 days), divergent results have been observed adding further complexity to the ROS signalling concept in HPV. Indeed, the authors demonstrated that total superoxide concentration, mitochondrial ROS and cellular $\mathrm{H}_{2} \mathrm{O}_{2}$ were reduced in PASMCs under chronic hypoxia, as seen in human pulmonary arterial hypertension (PAH) and other PAH models [15-17]. These changes were accompanied by a presumably adaptive augmentation of antioxidant enzymes expression and a similar level of the oxidative DNA damage marker, 8-hydroxyguanosine, which is also in accordance with a greater DNA repair capacity reported in PAH PASMCs [18-20]. Consistently, MitoQ had no effect on hypoxia-induced PASMC proliferation. Although effects of chronic hypoxia on isolated lung fibroblasts and particularly adventitial fibroblasts remained to be addressed, a trend towards lower superoxide levels was observed in lung homogenate of mice after chronic hypoxia $\left(10 \% \mathrm{O}_{2}\right.$ for 4 weeks). In accordance, MitoQ did not affect right ventricular systolic pressure and pulmonary vascular remodelling, both increased following chronic hypoxic exposure. However, the authors elegantly demonstrated in this model that MitoQ diminished superoxide concentration and attenuated RV remodelling. To further validate the functional importance of enhanced ROS production in RV dysfunction, hypoxia-independent RV hypertrophy was induced in mice by pulmonary artery banding. Similar to that observed in the chronic hypoxia model, MitoQ treatment significantly reduced RV superoxide concentration and improved RV remodelling. In agreement with this, previous reports have shown that antioxidants, such as EUK-134 and melatonin, decrease RV oxidative stress, attenuate the development of cardiomyocyte hypertrophy, and prevent RV fibrosis in the monocrotaline rat model of PAH [21, 22]. Together, these studies suggest that targeting oxidative stress could be an effective therapeutic strategy against RV dysfunction.

While the data generated from this study should be interpreted cautiously, they underscore a cellular adaptation to acute and chronic hypoxia with a cell-type dependent biphasic pattern of ROS production. Using expression of LDHA and PDK1 as read out of mitochondrial metabolism; the authors surmised that the metabolic reprogramming towards glycolysis undergone by PASMCs is the cause of the observed diminution of ROS levels in chronic hypoxia compared to acute hypoxia. Nevertheless, treatment of chronic hypoxia-exposed PASMCs with dichloroacetate (DCA), a compound that promotes mitochondrial oxidative metabolism and improves $\mathrm{PH}$ both in animal models and humans [23, 24], did not enhance mitochondrial superoxide concentration in cells exposed to chronic hypoxia, raising the hypothesis that DCA ameliorates $\mathrm{PH}$ by a superoxide-independent mechanism. However, findings in cancer cells showing that DCA reverses the Warburg effect, enhances generation of mitochondrial ROS and causes cell apoptosis do not support this hypothesis $[25,26]$. Interestingly, a divergent regulation of ROS production by PASMCs in acute and chronic hypoxia was previously reported with a significant decrease of ROS production upon acute exposure and a robust increase in ROS levels after chronic exposure [27]. Although additional work is needed to reconcile these discrepant experimental results, PAK et al. [14] have brought to light new evidence of the mutual relationship between hypoxia and ROS, fuelling the continuing debate surrounding redox events in pulmonary oxygen sensing. Interesting related questions remain. For instance, it is unclear which ROS change and how ROS vary between subcellular compartments and cell types and whether ROS production depends on the metabolic state of the cell.

\section{References}

1 Dunham-Snary KJ, Wu D, Sykes EA, et al. Hypoxic pulmonary vasoconstriction: from molecular mechanisms to medicine. Chest 2017; 151: 181-192.

2 Sommer N, Strielkov I, Pak O, et al. Oxygen sensing and signal transduction in hypoxic pulmonary vasoconstriction. Eur Respir J 2016; 47: 288-303.

3 Shimoda LA, Undem C. Interactions between calcium and reactive oxygen species in pulmonary arterial smooth muscle responses to hypoxia. Respir Physiol Neurobiol 2010; 174: 221-229.

4 Veit F, Pak O, Brandes RP, et al. Hypoxia-dependent reactive oxygen species signaling in the pulmonary circulation: focus on ion channels. Antioxid Redox Signal 2015; 22: 537-552.

5 Weir EK, Archer SL. The role of redox changes in oxygen sensing. Respir Physiol Neurobiol 2010; 174: $182-191$.

6 Archer SL, Huang J, Henry T, et al. A redox-based $\mathrm{O}_{2}$ sensor in rat pulmonary vasculature. Circ Res 1993; 73: 1100-1112. 
7 Michelakis ED, Thebaud B, Weir EK, et al. Hypoxic pulmonary vasoconstriction: redox regulation of $\mathrm{O}_{2}$-sensitive $\mathrm{K}+$ channels by a mitochondrial $\mathrm{O}_{2}$-sensor in resistance artery smooth muscle cells. J Mol Cell Cardiol 2004; 37: $1119-1136$.

8 Michelakis ED, Hampl V, Nsair A, et al. Diversity in mitochondrial function explains differences in vascular oxygen sensing. Circ Res 2002; 90: 1307-1315.

9 Waypa GB, Schumacker PT. Hypoxic pulmonary vasoconstriction: redox events in oxygen sensing. J Appl Physiol 2005; 98: 404-414

10 Waypa GB, Marks JD, Mack MM, et al. Mitochondrial reactive oxygen species trigger calcium increases during hypoxia in pulmonary arterial myocytes. Circ Res 2002; 91: 719-726.

11 Waypa GB, Guzy R, Mungai PT, et al. Increases in mitochondrial reactive oxygen species trigger hypoxia-induced calcium responses in pulmonary artery smooth muscle cells. Circ Res 2006; 99: 970-978.

12 Adesina SE, Kang BY, Bijli KM, et al. Targeting mitochondrial reactive oxygen species to modulate hypoxia-induced pulmonary hypertension. Free Radic Biol Med 2015; 87: 36-47.

13 Sheehan DW, Giese EC, Gugino SF, et al. Characterization and mechanisms of $\mathrm{H}_{2} \mathrm{O}_{2}$-induced contractions of pulmonary arteries. Am J Physiol 1993; 264: H1542-H1547.

14 Pak $\mathrm{O}$, Scheibe $\mathrm{S}$, Esfandiary $\mathrm{A}$, et al. Impact of the mitochondria-targeted antioxidant MitoQ on hypoxia-induced pulmonary hypertension. Eur Respir J 2018; 51: 1701024

15 Bonnet S, Michelakis ED, Porter CJ, et al. An abnormal mitochondrial-hypoxia inducible factor-1alpha-Kv channel pathway disrupts oxygen sensing and triggers pulmonary arterial hypertension in fawn hooded rats: similarities to human pulmonary arterial hypertension. Circulation 2006; 113: 2630-2641.

16 Sutendra G, Dromparis P, Wright $\mathrm{P}$, et al. The role of Nogo and the mitochondria-endoplasmic reticulum unit in pulmonary hypertension. Sci Transl Med 2011; 3: 88ra55.

17 Sutendra G, Bonnet S, Rochefort G, et al. Fatty acid oxidation and malonyl-CoA decarboxylase in the vascular remodeling of pulmonary hypertension. Sci Transl Med 2010; 2: 44ra58.

18 Meloche J, Le Guen M, Potus F, et al. miR-223 reverses experimental pulmonary arterial hypertension. Am J Physiol, Cell Physiol 2015; 309: C363-C372.

19 Meloche J, Pflieger A, Vaillancourt M, et al. Role for DNA damage signaling in pulmonary arterial hypertension. Circulation 2014; 129: 786-797.

20 Boucherat O, Peterlini T, Bourgeois A, et al. Mitochondrial HSP90 accumulation promotes vascular remodeling in pulmonary arterial hypertension. Am J Respir Crit Care Med 2018; in press [https://doi.org/10.1164/rccm. 201708-1751OC].

21 Maarman G, Blackhurst D, Thienemann F, et al. Melatonin as a preventive and curative therapy against pulmonary hypertension. J Pineal Res 2015; 59: 343-353.

22 Redout EM, van der Toorn A, Zuidwijk MJ, et al. Antioxidant treatment attenuates pulmonary arterial hypertension-induced heart failure. Am J Physiol Heart Circ Physiol 2010; 298: H1038-H1047.

23 Michelakis ED, McMurtry MS, Wu XC, et al. Dichloroacetate, a metabolic modulator, prevents and reverses chronic hypoxic pulmonary hypertension in rats: role of increased expression and activity of voltage-gated potassium channels. Circulation 2002; 105: 244-250.

24 Michelakis ED, Gurtu V, Webster L, et al. Inhibition of pyruvate dehydrogenase kinase improves pulmonary arterial hypertension in genetically susceptible patients. Sci Transl Med 2017; 9: eaao4583.

25 Bonnet S, Archer SL, Allalunis-Turner J, et al. A mitochondria-K+ channel axis is suppressed in cancer and its normalization promotes apoptosis and inhibits cancer growth. Cancer Cell 2007; 11:37-51.

26 Kinnaird A, Dromparis P, Saleme B, et al. Metabolic modulation of clear-cell renal cell carcinoma with dichloroacetate, an inhibitor of pyruvate dehydrogenase kinase. Eur Urol 2016; 69: 734-744.

$27 \mathrm{Wu}$ W, Platoshyn O, Firth AL, et al. Hypoxia divergently regulates production of reactive oxygen species in human pulmonary and coronary artery smooth muscle cells. Am J Physiol Lung Cell Mol Physiol 2007; 293: L952-L959. 